\title{
Exploring Results Organisation for Image Searching
}

\author{
Jana Urban and Joemon M. Jose \\ Department of Computing Science, University of Glasgow, Glasgow G12 8RZ, UK \\ $\{$ jana, jj\}@dcs.gla.ac.uk
}

\begin{abstract}
An explorative study of an image retrieval interface with respect to the support it offers the user to organise their search results is presented. The evaluation, involving design professionals performing practical and relevant tasks, shows that the proposed approach succeeds in encouraging the user to conceptualise their tasks better.
\end{abstract}

\section{Introduction and Motivation}

Content-based image retrieval (CBIR) systems have still not managed to find favour with the public even after more than a decade of research effort. There are two main reasons for their lack of acceptability: first, the images' low-level feature representation does not reflect the high-level concepts the user has in mind (semantic gap) [1]; and - partially due to this - the user tends to have major difficulties in formulating and communicating their information need effectively (query formulation problem) 2. Moreover, current interfaces are limited to providing query facilities and result presentation. Our approach, in contrast, encourages the user to group and organise their search results and thus provide more fine-grained feedback for the system. It combines the search and management process, which - according to our hypothesis - helps the user to conceptualise their search tasks and to overcome the query formulation problem. The system assists the user by recommending relevant images for selected groups. This way, the user can concentrate on solving specific tasks rather than having to think about how to create a good query in accordance with the retrieval mechanism. In this paper we explore how useful the organisation of search results is for the user to solve their work tasks.

\section{The EGO Interface}

The EGO system is an image management and retrieval tool that learns from and adapts to a user by the way they interact with the image collection. A workspace is provided in the interface allowing the user to organise their search results. Images can be dragged onto the workspace and organised into groups. The grouping can be achieved in an interactive fashion with the help of a recommendation system. For a selected group, the system can recommend new images based on their similarity with the images already in the group. The user then has 


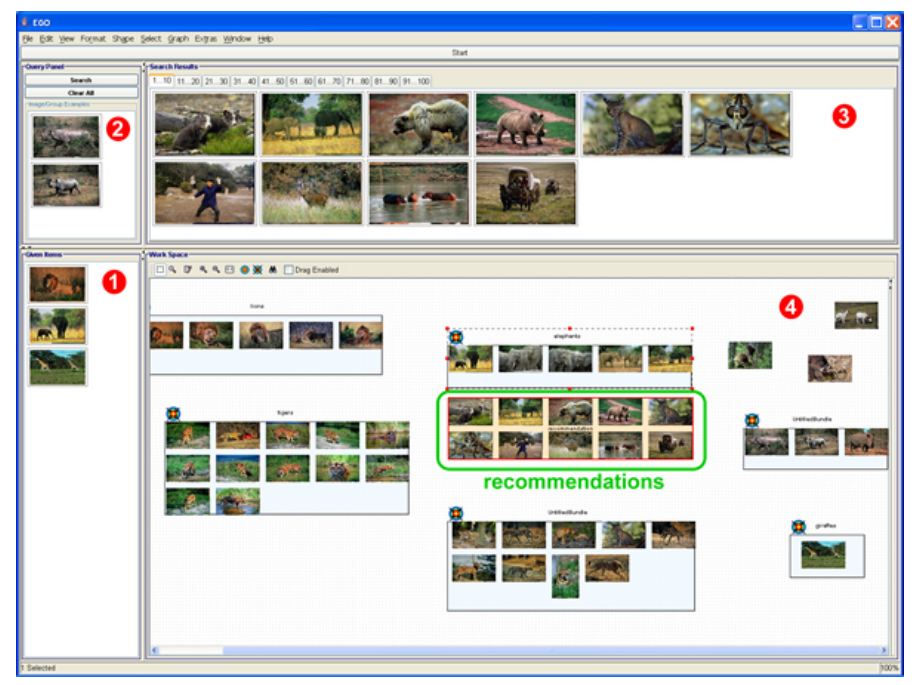

Fig. 1. Annotated EGO interface

the option of accepting any of the recommended images by dragging them into the group. An image can belong to multiple groups simultaneously. The query facilities available in the EGO interface are: (1) manually constructed queries by providing one or more image examples (QBE), and (2) user-requested recommendations. The underlying retrieval system is described in [3] and [4]. The learning strategy involves calculating an ideal query and the parameters of the matching function based on the provided images. The interface depicted in Figure 1 comprises the following components:

1. Given Items Panel contains a selection of images (three per task) provided for illustration purposes and that can be used to bootstrap the search;

2. $Q B E$ Panel provides a basic query facility by allowing the user to compose a search request by adding example images to this panel.

3. Results Panel displays the search results from a query constructed in the QBE panel. Any result image can be dragged onto the workspace to start organising the collection or into the QBE panel to change the current query.

4. Workspace Panel serves as the organisation ground for the user. Also, the recommendations will be displayed close to the selected group on the workspace.

\section{Experimental Methodology}

This study's objective is to analyse how people make use of the workspace, depending on the task nature, in order to judge the workspace's usefulness for helping the user to conceptualise their task. We conducted a task-oriented, usercentred evaluation [5], employing a collection of 12800 photographs (CD 1, CDs 4-6 of the Corel 1.6M dataset). 
Our sample user population consisted of post-graduate design students and young design professionals (20-30 years; 9 male, 3 female). Responses to an entry questionnaire indicated that our participants could be assumed to have a good understanding of the tasks we were to set them, but a more limited knowledge or experience of the search process.

We adopted a simulated work task situation [6], which allows the users to evolve their information needs in the same dynamic manner as they might do so in their real working lives. We have created two different task scenarios: the category search scenario and the design task scenario. By analysing the number of groups created and the number of images per group for the various tasks, we can identify how these numbers relate to task complexity. The participants were presented with the following work task scenario and task description:

Task Scenario Imagine you are a designer with responsibility for the design of leaflets on various subjects for the Wildlife Conservation (WLC). The leaflets are intended to raise awareness among the general public for endangered species and the preservation of their habitats. These leaflets [...] consisting of a body of text interspersed with up to 4-5 images selected on the basis of their appropriateness to the use to which the leaflets are put.

Category Search Task: You will be given a leaflet topic [...] Your task involves searching for as many images as you are able to find on the given topic, suitable for presentation in the leaflet. [...] You have 10 minutes to attempt this task.

Design Task: [...] you're asked to select images for a leaflet for WLC presenting the organisation and a selection of their activities [...] Your task is to search for suitable images and then make a pre-selection of 3-5 images for the leaflet. (20 minutes)

\section{Results Analysis}

Concerning the number of groups created in the design task, we could identify two different types of behaviour. About half the people saved all candidates on the workspace organised in 4-9 groups reflecting different aspects of the task before making the final selection. The other half only added a small number of images, mostly all in the same group. The average number of images saved on the workspace for the first selection strategy was 53 images in 6.5 groups. On the other hand, the other group of users saved only 14 images in 1.5 groups.

It is also interesting to highlight differences in behaviour in the design task and the category search task. The average number of groups per task is shown in Table 1. We can clearly see a dependency between the number of groups and the nature of the task. Tasks 1-3 are very focused (e.g. "Mountainous landscapes"), while Tasks 4-6 are composite/multi-faceted (e.g. "African Wildlife").

Table 1. Average Number of groups created per task

\begin{tabular}{|l|ccccc|c|c|}
\hline & Task 1 & Task & 2 & Task & 3 & Task & 4 \\
\hline
\end{tabular}


In addition, the questionnaire data points to differences in user perception depending on the task nature. The responses suggest they had a clearer idea of the images relevant for the task in the category search scenario (average 4.4, on a scale from 1-5), compared to the design scenario (3.7). However, the organisation of images into groups seems to be more helpful in the design scenario than in the category search scenario. The average of the responses to the statement, whether the system organisation of images into groups helps them express different aspects of the task, is 4.42 and 3.92 for the design task and category search task, respectively. The difference is even more pronounced comparing the different task groups for the category search tasks. The average response is 3.0 for the focused tasks and 4.83 for the more complex tasks. So, while the organisation is helpful in general, it is dependent on, and reflects the nature of, the task.

\section{Conclusion}

In this study, we found a correlation between the number of groups created and the complexity of the task set. Further, user responses showed that the management of search results was more helpful in the design scenario, which is more flexible and open to interpretation than the category search scenario. In the latter, the usefulness of the organisation also depended on the task's complexity: the more facets the task comprised, the more useful the workspace was considered. The dependency between both the number of groups created and the users' perception of the workspace's usefulness, led us to the conclusion that our approach indeed helps in conceptualising the task better.

In the future, the user should be assisted in determining task aspects and create groups (semi-) automatically. For a multi-aspect task, we could then group results into the various aspects and present recommendations for each group. The category task aims at maximising recall, while the design task aims at finding a selection of good quality images that work well together. The interface should have a way to be tailored to these contrasting requirements to adapt to its users.

\section{References}

1. Smeulders, A.W., Worring, M., Santini, S., Gupta, A., Jain, R.: Content-based image retrieval at the end of the early years. IEEE Trans. Pattern Analysis and Machine Intelligence 22 (2000) 1349-1380

2. ter Hofstede, A.H.M., Proper, H.A., van der Weide, T.P.: Query formulation as an information retrieval problem. The Computer Journal 39 (1996) 255-274

3. Urban, J., Jose, J.M.: EGO: A personalised multimedia management tool. In: Proc. of the 2nd Int. Workshop on Adaptive Multimedia Retrieval. (2004) 3-17

4. Urban, J., Jose, J.M.: Evidence combination for multi-point query learning in content-based image retrieval. In: Proc. of the IEEE 6th Int. Symposium on Multimedia Software Engineering (MSE 2004). (2004) 583-586

5. Ingwersen, P.: Information Retrieval Interaction. Taylor Graham, London (1992)

6. Jose, J.M., Furner, J., Harper, D.J.: Spatial querying for image retrieval: A useroriented evaluation. In: Proc. of the ACM SIGIR Conf. (1998) 232-240 